The Astrophysical Journal, 664:L87-L90, 2007 August 1

(C) 2007. The American Astronomical Society. All rights reserved. Printed in U.S.A.

\title{
DISCOVERY OF VERY HIGH ENERGY GAMMA RADIATION FROM IC 443 WITH THE MAGIC TELESCOPE
}

J. Albert, ${ }^{1}$ E. Aliu,${ }^{2}$ H. Anderhub,${ }^{3}$ P. Antoranz,${ }^{4}$ A. Armada, ${ }^{2}$ C. Baixeras, ${ }^{5}$ J. A. Barrio, ${ }^{4}$ H. Bartko, ${ }^{6,7}$ D. Bastieri,${ }^{8}$ J. K. Becker, ${ }^{9}$ W. Bednarek, ${ }^{10}$ K. Berger, ${ }^{1}$ C. Bigongiari,${ }^{8}$ A. Biland, ${ }^{3}$ R. K. Bock,${ }^{6,8}$ P. Bordas, ${ }^{11}$ V. Bosch-Ramon, ${ }^{11}$

T. Bretz, ${ }^{1}$ I. Britvitch, ${ }^{3}$ M. Camara, ${ }^{4}$ E. Carmona, ${ }^{6}$ A. Chilingarian, ${ }^{12}$ J. A. Coarasa, ${ }^{6}$ S. Commichau, ${ }^{3}$

J. L. Contreras, ${ }^{4}$ J. Cortina, ${ }^{2}$ M. T. Costado, ${ }^{13,14}$ V. Curtef, ${ }^{9}$ V. Danielyan, ${ }^{12}$ F. Dazzi, ${ }^{8}$ A. De Angelis, ${ }^{15}$

C. Delgado, ${ }^{7,13}$ R. De los Reyes, ${ }^{4}$ B. De Lotto, ${ }^{15}$ E. Domingo-Santamaría, ${ }^{2}$ D. Dorner, ${ }^{1}$ M. Doro, ${ }^{8}$ M. Errando, ${ }^{2}$

M. Fagiolini, ${ }^{16}$ D. Ferenc, ${ }^{17}$ E. Fernández, ${ }^{2}$ R. Firpo, ${ }^{2}$ J. Flix, ${ }^{2}$ M. V. Fonseca, ${ }^{4}$ L. Font,${ }^{5}$ M. Fuchs, ${ }^{6}$ N. Galante, ${ }^{6}$

R. J. García-López, ${ }^{13,14}$ M. GarczarczyK, ${ }^{6}$ M. Gaug, ${ }^{13}$ M. Giller, ${ }^{10}$ F. Goebel, ${ }^{6}$ D. HaKobyan, ${ }^{12}$ M. Hayashida, ${ }^{6}$

T. Hengstebeck, ${ }^{18}$ A. Herrero, ${ }^{13,14}$ D. Höhne, ${ }^{1}$ J. Hose, ${ }^{6}$ C. C. Hsu, ${ }^{6}$ P. Jacon, ${ }^{10}$ T. Jogler, ${ }^{6}$ R. Kosyra, ${ }^{6}$ D. Kranich, ${ }^{3}$

R. Kritzer ${ }^{1}$ A. Laille, ${ }^{17}$ E. Lindfors, ${ }^{19}$ S. Lombardi, ${ }^{8}$ F. Longo, ${ }^{15}$ J. López, ${ }^{2}$ M. López, ${ }^{4}$ E. Lorenz, ${ }^{3,6}$ P. Majumdar, ${ }^{6}$ G. Maneva, ${ }^{20}$ K. Mannheim, ${ }^{1}$ O. Mansutti,${ }^{15}$ M. Mariotti,${ }^{8}$ M. Martínez, ${ }^{2}$ D. Mazin, ${ }^{2}$ C. MercK, ${ }^{6}$ M. Meucci, ${ }^{16}$ M. Meyer, ${ }^{1}$ J. M. Miranda, ${ }^{4}$ R. Mirzoyan, ${ }^{6}$ S. Mizobuchi,${ }^{6}$ A. Moralejo, ${ }^{2}$ D. Nieto, ${ }^{4}$ K. Nilsson, ${ }^{19}$ J. Ninkovic,${ }^{6}$ E. Oña-Wilhelmi, ${ }^{2}$ N. Otte, ${ }^{6,18}$ I. Oya, ${ }^{4}$ D. Paneque, ${ }^{6}$ M. Panniello, ${ }^{13,21}$ R. Paoletti, ${ }^{16}$ J. M. Paredes, ${ }^{11}$ M. Pasanen, ${ }^{19}$ D. Pascoli,${ }^{8}$ F. Pauss,${ }^{3}$ R. Pegna,${ }^{16}$ M. Persic, ${ }^{15,22}$ L. Peruzzo,${ }^{8}$ A. Piccioli, ${ }^{16}$ E. Prandini, ${ }^{8}$ N. Puchades, ${ }^{2}$ A. Raymers, ${ }^{12}$ W. Rhode, ${ }^{9}$ M. Ribó, ${ }^{11}$ J. Rico, ${ }^{2}$ M. Rissi, ${ }^{3}$ A. Robert, ${ }^{5}$ S. Rügamer, ${ }^{1}$ A. Saggion, ${ }^{8}$ T. Saito, ${ }^{6}$ A. Sánchez, ${ }^{5}$ P. Sartori, ${ }^{8}$ V. Scalzotto, ${ }^{8}$ V. Scapin, ${ }^{15}$ R. Schmitt, ${ }^{1}$ T. Schweizer, ${ }^{6}$ M. Shayduk, ${ }^{6,18}$ K. Shinozaki, ${ }^{6}$ S. N. Shore, ${ }^{23}$ N. Sidro, ${ }^{2}$ A. Sillanpää, ${ }^{19}$ D. Sobczynska, ${ }^{10}$ A. Stamerra, ${ }^{16}$ L. S. Stark, ${ }^{3}$ L. Takalo, ${ }^{19}$ P. Temnikov, ${ }^{20}$ D. Tescaro, ${ }^{2}$ M. Teshima, ${ }^{6}$ D. F. Torres, ${ }^{24}$ N. Turini, ${ }^{16}$ H. Vankov, ${ }^{20}$ V. Vitale, ${ }^{15}$ R. M. Wagner, ${ }^{6}$ T. Wibig, ${ }^{10}$ W. WitteK, ${ }^{6}$ F. Zandanel, ${ }^{8}$ R. ZANIN, ${ }^{2}$ AND J. ZAPATERO ${ }^{5}$

Received 2007 May 21; accepted 2007 June 19; published 2007 July 17

\section{ABSTRACT}

We report the detection of a new source of very high energy (VHE; $E_{\gamma} \geq 100 \mathrm{GeV}$ ) $\gamma$-ray emission located close to the Galactic plane, MAGIC J0616+225, which is spatially coincident with supernova remnant IC 443. The observations were carried out with the MAGIC telescope in the periods 2005 December-2006 January and 2006 December-2007 January. Here we present results from this source, leading to a VHE $\gamma$-ray signal with a statistical significance of $5.7 \sigma$ in the 2006/2007 data and a measured differential $\gamma$-ray flux consistent with a power law, described as $d N_{\gamma} /(d A d t d E)=(1.0 \pm 0.2) \times 10^{-11}(E / 0.4 \mathrm{TeV})^{-3.1 \pm 0.3} \mathrm{~cm}^{-2} \mathrm{~s}^{-1} \mathrm{TeV}^{-1}$. We briefly discuss the observational technique used and the procedure implemented for the data analysis. The results are placed in the context of the multiwavelength emission and the molecular environment found in the region of IC 443.

Subject headings: gamma rays: observations - ISM: individual (IC 443, MAGIC J0616+225) supernova remnants

\section{INTRODUCTION}

IC 443 is an asymmetric shell-type supernova remnant (SNR) with a diameter of $45^{\prime}$ at a distance of about $1.5 \mathrm{kpc}$ (Fesen 1984; Claussen et al. 1997). It is included in Green's catalog (Green 2004), and it has a spectral index of 0.36 and a flux density of $160 \mathrm{Jy}$ at $1 \mathrm{GHz}$. It was mapped in radio with the VLA at $90 \mathrm{~cm}$ (Claussen et al. 1997) and also at 20,6, and $3.5 \mathrm{~cm}$ (Olbert et al. 2001; Condon et al. 1998). Moreover, Claussen et al. (1997) reported the presence of maser emission at $1720 \mathrm{MHz}$ from four sources, the strongest of which is located at $(l, b) \sim\left(-171.0^{\circ}\right.$,

${ }^{1}$ Universität Würzburg, D-97074 Würzburg, Germany.

${ }^{2}$ IFAE, Edifici Cn., E-08193 Bellaterra (Barcelona), Spain.

${ }^{3}$ ETH Zurich, CH-8093 Switzerland.

${ }^{4}$ Universidad Complutense, E-28040 Madrid, Spain.

${ }^{5}$ Universitat Autònoma de Barcelona, E-08193 Bellaterra, Spain.

${ }^{6}$ Max-Planck-Institut für Physik, D-80805 München, Germany.

${ }^{7}$ Correspondence: H. Bartko (hbartko@mppmu.mpg.de) and C. Delgado (delgadom@iac.es).

${ }^{8}$ Università di Padova and INFN, I-35131 Padova, Italy.

${ }^{9}$ Universität Dortmund, D-44227 Dortmund, Germany.

${ }^{10}$ University of Łódź, PL-90236 Lodz, Poland.

${ }^{11}$ Universitat de Barcelona, E-08028 Barcelona, Spain.

${ }^{12}$ Yerevan Physics Institute, AM-375036 Yerevan, Armenia. $\left.2.9^{\circ}\right)$. IC 443 is a prominent X-ray source, with data available from ROSAT (Asaoka \& Aschenbach 1994), ASCA (Keohane et al. 1997), XMM (Bocchino \& Bykov 2000, 2001, 2003; Bykov et al. 2005; Troja et al. 2006), and Chandra (Olbert et al. 2001; Gaensler et al. 2006). The EGRET has detected a $\gamma$-ray source (3EG J0617+2238) above $100 \mathrm{MeV}$ in IC 443 (Esposito et al. 1996; Hartman et al. 1999). Upper limits to the very high energy (VHE) $\gamma$-ray emission from IC 443 were reported by the Whipple collaboration, $d N_{\gamma} /(d A d t)<6 \times 10^{-12} \mathrm{~cm}^{-2} \mathrm{~s}^{-1}(0.11 \mathrm{crab})$ above $500 \mathrm{GeV}$ (Holder et al. 2005), and by the CAT collaboration, $d N_{\gamma} /(d A d t)<9 \times 10^{-12} \mathrm{~cm}^{-2} \mathrm{~s}^{-1}$ above $250 \mathrm{GeV}$ (Khelifi 2003).

Here we present observations of the SNR IC 443 with the

\footnotetext{
${ }^{13}$ Instituto de Astrofisica de Canarias, E-38200, La Laguna, Tenerife, Spain.

${ }^{14}$ Departamento de Astrofísica, Universidad de La Laguna, E-38206 La Laguna, Tenerife, Spain.

${ }^{15}$ Università di Udine and INFN Trieste, I-33100 Udine, Italy.

${ }^{16}$ Università di Siena and INFN Pisa, I-53100 Siena, Italy.

${ }^{17}$ University of California, Davis, CA-95616-8677.

${ }^{18}$ Humboldt-Universität zu Berlin, D-12489 Berlin, Germany.

${ }^{19}$ Tuorla Observatory, Turku University, FI-21500 Piikkiö, Finland.

${ }^{20}$ Institute for Nuclear Research and Nuclear Energy, BG-1784 Sofia, Bulgaria.

${ }^{21}$ Deceased.

${ }^{22}$ INAF/Osservatorio Astronomico and INFN, I-34131 Trieste, Italy.

${ }^{23}$ Università di Pisa and INFN Pisa, I-56126 Pisa, Italy.

${ }^{24}$ ICREA and Institut de Cienciès de l'Espai (IEEC-CSIC), E-08193 Bellaterra, Spain.
} 


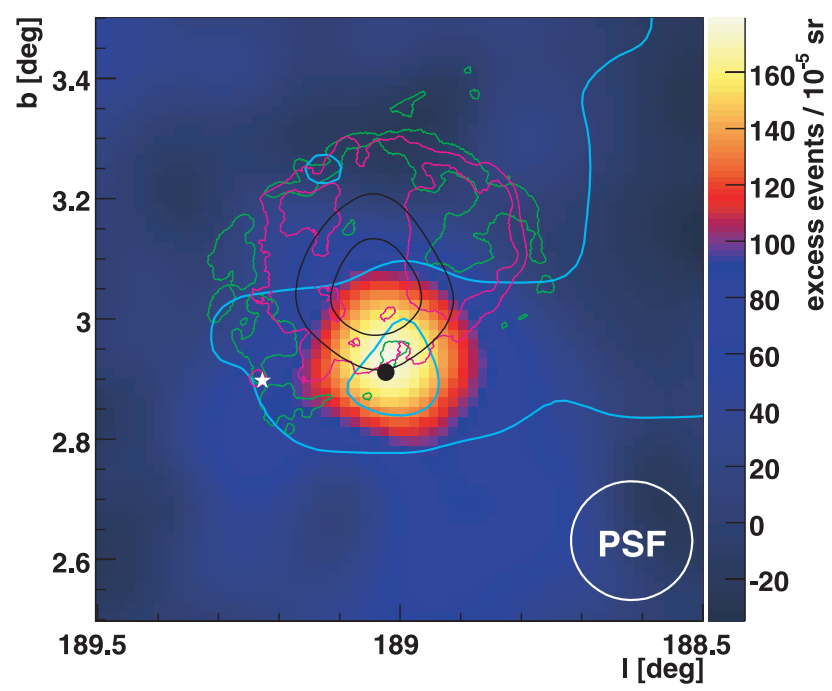

FIG. 1.-Sky map of $\gamma$-ray candidate events (background-subtracted) in the direction of MAGIC J0616+225 for an energy threshold of about $150 \mathrm{GeV}$ in Galactic coordinates. Overlaid are ${ }^{12} \mathrm{CO}$ emission contours (cyan) from Dame et al. (2001), contours of $20 \mathrm{~cm}$ VLA radio data from Condon et al. (1998; green), X-ray contours from ROSAT (purple; Asaoka \& Aschenbach 1994), and $\gamma$-ray contours from EGRET (black; Hartman et al. 1999). The ${ }^{12} \mathrm{CO}$ contours are at 7 and $14 \mathrm{~K} \mathrm{~km} \mathrm{~s}^{-1}$, integrated from -20 to $20 \mathrm{~km} \mathrm{~s}^{-1}$ in velocity, the range that best defines the molecular cloud associated with IC 443. The contours of the radio emission are at $5 \mathrm{mJy} \mathrm{beam}^{-1}$, chosen for showing the SNR IC 443. The X-ray contours are at 700 and 1200 counts per $6 \times 10^{-7}$ sr. The EGRET contours represent a $68 \%$ and $95 \%$ statistical probability that a single source lies within the given contour. The white star denotes the position of the pulsar CXOU J061705.3+222127 (Olbert et al. 2001). The black circle shows the position of the $1720 \mathrm{MHz} O H$ maser (Claussen et al. 1997). The white circle shows the MAGIC PSF of $\sigma=0.1^{\circ}$.

Major Atmospheric Gamma Imaging Cerenkov (MAGIC) telescope that resulted in the detection of a new source of VHE $\gamma$-rays, which we named MAGIC J0616+225.

\section{OBSERVATIONS}

MAGIC (see, e.g., Baixeras et al. 2004 and Cortina et al. 2005 for a detailed description) is the largest single-dish imaging air Cerenkov telescope (IACT) in operation. ${ }^{25}$ It is located on the Canary Island La Palma $\left(28.8^{\circ} \mathrm{N}, 17.8^{\circ} \mathrm{W}, 2200 \mathrm{~m}\right.$ above sea level).

The SNR IC 443 was observed for a total of $10 \mathrm{hr}$ in the period 2005 December-2006 January (period I), with the telescope pointing to the SNR center. The VHE $\gamma$-ray sky map showed evidence of a VHE $\gamma$-ray signal with a statistical significance of $3 \sigma$ (before trials were taken into account). To test the hypothesis that this excess is due to a VHE $\gamma$-ray source, MAGIC J0616 +225, the excess center was observed for a total of $37 \mathrm{hr}$ in the period 2006 December-2007 January (period II). Therefore, in the analysis of the period II data, no trial factors needed to be taken into account to compute the statistical significance of the source detection. Changes in the readout chain in spring 2006 due to the installation of a novel 2 Gsamples s $^{-1}$ FADC system (Bartko et al. 2005; Goebel et al. 2007) in parallel to the existing 300 Msamples $\mathrm{s}^{-1}$ FADC system made it advisable (on grounds of simplicity of the analysis) to

\footnotetext{
${ }^{25}$ For a technical description, see also http://wwwmagic.mppmu.mpg.de/ magic/factsheet/.
}

select only the second part of the data set, period II, for the studies presented here. However, within statistics the data from period I provide compatible results.

At La Palma, IC 443 culminates at about $6^{\circ}$ zenith angle (ZA). The observations were carried out in the false-source tracking (wobble) mode (Fomin et al. 1994). The sky directions (W1, W2) to be tracked were on two opposite sides of the source direction, at a distance of $0.4^{\circ}$ from the source. They were chosen such that in the camera, the sky brightness distribution relative to $\mathrm{W} 1$ was similar to the one relative to $\mathrm{W} 2$. For each tracking position, three circular background control regions were defined, located symmetrically to the source region with respect to the camera center. During wobble mode data taking, $50 \%$ of the data was taken at W1, and 50\% at W2, switching (wobbling) between the two directions every 20 minutes. In total, about 8 million triggers were recorded in period I, and about 30 million triggers were recorded in period II. There are two bright stars in the field of view: $\eta$ Gem $(V=3.28$ and $B-V=1.60)$ at a distance to MAGIC J0616+225 of $0.4^{\circ}$ and $\mu$ Gem $(V=2.88$ and $B-$ $V=1.64$ ) at a distance to MAGIC J0616+225 of $1.9^{\circ}$. Both stars are rather red such that the increase in the pixel anode currents is still sustainable at nominal pixel high voltage (Albert et al. 2007a; Paneque et al. 2004). The discriminator thresholds of the channels included in the trigger are dynamically regulated to keep the individual pixel rates at a constant level (Cortina et al. 2005).

\section{DATA ANALYSIS}

Data runs with anomalous trigger rates due to bad observation conditions (three nights of data taking) were rejected from further analysis. In addition, data with $\mathrm{ZA}>30^{\circ}(2 \mathrm{hr})$ were excluded. The remaining data set corresponded to an effective observation time (including a dead-time correction) of $29 \mathrm{hr}$.

The data analysis was carried out using the standard MAGIC analysis and reconstruction software (Bretz \& Wagner 2003), the first step of which involves the FADC signal reconstruction using a digital filter and the calibration of the raw data (Albert et al. 2006d; Gaug et al. 2005). Thereafter, shower images were cleaned by applying a cut of 10 photoelectrons for image core pixels and 5 photoelectrons for boundary pixels (see, e.g., Fegan 1997). These tail cuts were scaled for the larger size of the outer pixels of the MAGIC camera. The camera images were characterized by image parameters (Hillas 1985). In this analysis, the Random Forest method (see Bock et al. 2004 and Breiman 2001 for a detailed description) was applied for the $\gamma /$ hadron separation (for a review see, e.g., Fegan 1997) as well as for the energy estimation (see, e.g., Albert et al. 2006a, 2006b, 2006c, 2007b). Above the analysis energy threshold, the cut efficiency reaches about $50 \%$, corresponding to an effective collection area for $\gamma$-ray showers of about $50000 \mathrm{~m}^{2}$.

For each event, the arrival direction of the primary $\gamma$-ray candidate in sky coordinates was estimated using the DISP method (Fomin et al. 1994; Lessard et al. 2001; Domingo-Santamaria et al. 2005). A conservative cut of SIZE $\geq 200$ photoelectrons was applied to select a subset of events with superior angular resolution. For these events, the effect of the bright stars in the field of view is negligible. The corresponding analysis energy threshold (the energy corresponding to the maximum of the differential $\gamma$-ray rate after all analysis cuts) was about $150 \mathrm{GeV}$.

Figure 1 shows the sky map of $\gamma$-ray candidates (backgroundsubtracted) from the direction of MAGIC J0616+225 in Galactic coordinates. It was folded with a two-dimensional Gaussian with a standard deviation of $0.072^{\circ}$. The MAGIC $\gamma$-ray point-spread 


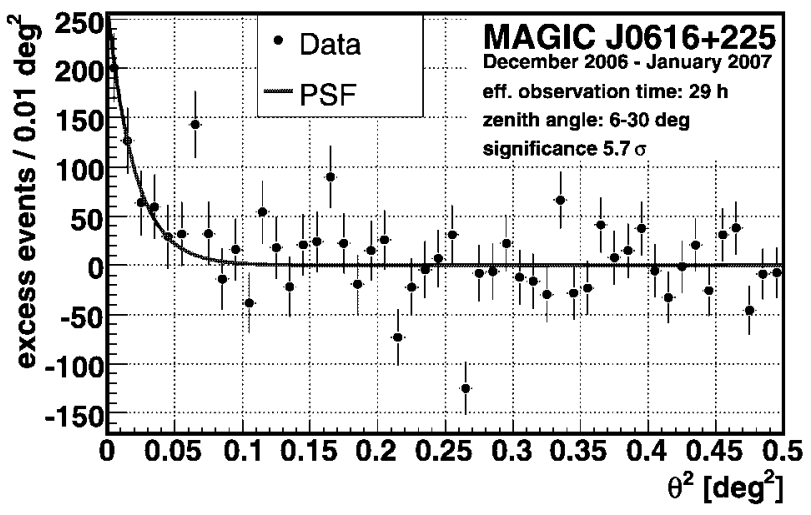

FIG. 2.-Distribution of excess $\gamma$-ray candidate events (Fig. 1) as a function of the squared angular distance from the excess center of MAGIC J0616+225 (points), compared to the expected distribution for a pointlike source (blue solid line) corresponding to the MAGIC PSF.

function (PSF; standard deviation of a two-dimensional Gaussian fit to the nonfolded brightness profile of a point source) is $0.10^{\circ} \pm 0.01^{\circ}$ for SIZE $\geq 200$ photoelectrons (Domingo-Santamaria et al. 2005). The sky map is overlaid with contours of ${ }^{12} \mathrm{CO}$ emission (cyan) from Dame et al. (2001), contours of $20 \mathrm{~cm}$ VLA radio data from Condon et al. (1998; green), X-ray contours from ROSAT (purple; Asaoka \& Aschenbach 1994), and $\gamma$-ray contours from EGRET (black; Hartman et al. 1999). Also, XMM-Newton and Chandra X-ray observations of IC 443 exist; they will be discussed below. The white star denotes the position of the pulsar CXOU J061705.3+222127 (Olbert et al. 2001). The black circle shows the position of the $1720 \mathrm{MHz} \mathrm{OH}$ maser (Claussen et al. 1997). The VHE $\gamma$-ray sky map shows a clear excess centered at (R.A., decl.) $=\left(06^{\mathrm{h}} 16^{\mathrm{m}} 43^{\mathrm{s}},+22^{\circ} 31^{\prime} 48^{\prime \prime}\right)$, i.e., at MAGIC $\mathrm{J} 0616+225$. The statistical position error is $1.5^{\prime}$, and the systematic error due to background determination and pointing uncertainty is estimated to be $1^{\prime}$ (Albert et al. 2007b). Within errors, MAGIC J0616 +225 is pointlike. The center of gravity of the excess in the period II data shown here agrees with the center of gravity of the excess in the period I data not shown here.

Figure 2 shows the distribution of the squared angular distance, $\theta^{2}$, with respect to the excess center, together with the expected distribution for a pointlike source, for the excess $\gamma$-ray-like events in Figure 1. Choosing a conservative $\theta^{2}$ cut of $\theta^{2} \leq$ $0.05 \mathrm{deg}^{2}$, as appropriate for unidentified sources, we find that the observed excess in the period II data set in the direction of MAGIC J0616+225 has a significance of $5.7 \sigma$. The period I data showed an excess of about $3 \sigma$ significance at a position less than $3^{\prime}=0.05^{\circ}$ away from period II data set. Computing the significance of the period II data set with respect to the period I position yields only a negligible difference for the chosen large signal region $\theta^{2} \leq 0.05 \mathrm{deg}^{2}$. Choosing smaller $\theta^{2}$ cuts, one obtains a higher significance for the period II excess center.

For the spectral analysis, the excess data from a sky region with a maximum angular distance of $\theta^{2}=0.05 \mathrm{deg}^{2}$ around the excess center were integrated. Figure 3 shows the reconstructed VHE $\gamma$-ray spectrum $\left[d N_{\gamma} /\left(d E_{\gamma} d A d t\right)\right.$ vs. true $\left.E_{\gamma}\right]$ of MAGIC J0616+225 after correcting (unfolding) for the instrumental energy resolution (Anykeev et al. 1991; Bertero 1989). The horizontal bars indicate the bin size in energy. A simple power law was fit to the spectral points, taking into account correlations between the spectral points that are introduced by the unfolding procedure. The result is given by $\left(\chi^{2} /\right.$ dof $\left.=1.1\right) d N_{\gamma} /(d A d t d E)=(1.0 \pm 0.2) \times 10^{-11}(E / 0.4$

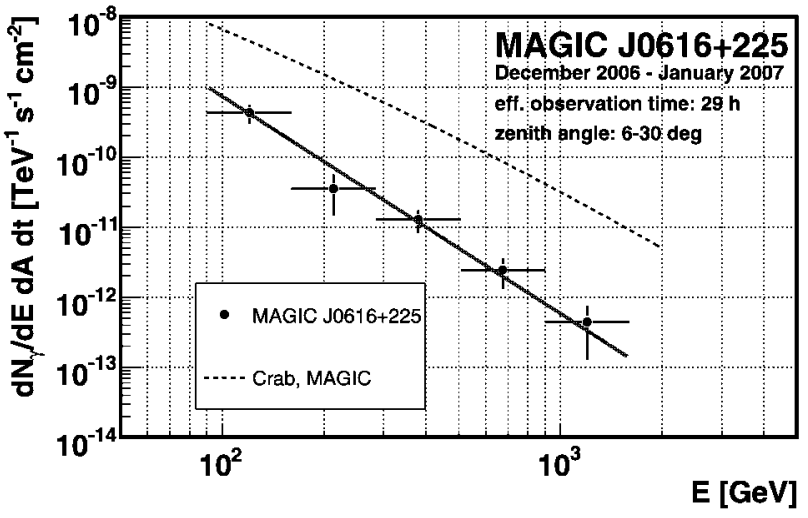

FIG. 3.-VHE $\gamma$-ray spectrum of MAGIC J0616+225 (statistical errors only). The blue solid line shows the result of a simple power law fit to the spectral points, taking into account correlations between the spectral points that are introduced by the unfolding procedure. The dotted line shows the spectrum of the Crab Nebula as measured by MAGIC (Albert et al. 2007b).

$\mathrm{TeV})^{-3.1 \pm 0.3} \mathrm{~cm}^{-2} \mathrm{~s}^{-1} \mathrm{TeV}^{-1}$. The quoted errors are statistical. The systematic error is estimated to be $35 \%$ in the flux level determination and 0.2 in the spectral index (see also Albert et al. 2006b, 2007b). The integral flux of MAGIC J0616+225 above $100 \mathrm{GeV}$ is about $6.5 \%$ of the Crab Nebula flux, and above $300 \mathrm{GeV}$, it is about $2.8 \%$ of the Crab Nebula flux. The integral flux of MAGIC J0616+225 in the observation period II is compatible within errors with that of period I. Within the two observation periods ( 2 months each), no flux variations exceeding the measurement errors were observed. All data analysis steps were cross-checked by a second, independent analysis, yielding compatible results.

\section{DISCUSSION AND CONCLUDING REMARKS}

If, due to the spatial association shown in Figure 1, we accept that MAGIC J0616+225 is associated with SNR IC 443, located at a distance of $\sim 1.5 \mathrm{kpc}$, then it has a luminosity between $100 \mathrm{GeV}$ and $1 \mathrm{TeV}$ of about $2.7 \times 10^{33} \mathrm{ergs} \mathrm{s}^{-1}$. This is roughly 1 order of magnitude below the luminosity of the sources HESS J1813-178 and J1834-087 (Aharonian et al. 2006), both also detected by MAGIC (Albert et al. 2006a, 2006c). In addition, the $\gamma$-ray spectrum of MAGIC J0616+225 is steeper than those of $\mathrm{J} 1813-178$ and $\mathrm{J} 1834-087$.

Figure 1 indeed shows a very interesting multifrequency phenomenology. The MAGIC VHE $\gamma$-ray source is slightly displaced with respect to the central position of the EGRET source 3EG J0617+2238, although still consistent with it, within errors. An independent analysis of $\mathrm{GeV}$ photons measured by EGRET resulted in the source GeV J0617+2237 (Lamb \& Macomb 1997) being in the same location as 3EG J0617+2238. Gaisser et al. (1998), Baring et al. (1999), and Kaul (2001) extrapolate the energy spectrum of 3EG J0617+2238 into the VHE $\gamma$-ray range. The predicted fluxes are higher, with a harder energy spectrum than that of MAGIC J0616+225.

The EGRET source is located in the center of the SNR, whereas the VHE $\gamma$-ray source is displaced to the south, in direct correlation with a molecular cloud. The molecular cloud environment surrounding IC 443 and its possible connection with the EGRET $\gamma$-ray source were studied by Torres et al. (2003). There is a large amount of molecular mass $\left(\$ 10^{4} M_{\odot}\right)$, consistent with the distance to the SNR IC 443, corresponding to a velocity range of -20 to $20 \mathrm{~km} \mathrm{~s}^{-1}$, as shown in Figure 1. The highest $\mathrm{CO}$ intensity detected is directly superimposed on the central 
position of the MAGIC source. Moreover, Claussen et al. (1997) reported the presence of maser emission from $(l, b) \sim\left(-171.0^{\circ}\right.$, $2.9^{\circ}$ ), spatially correlated with the MAGIC source (see also Hewitt et al. 2006). The maser emission is an indication of a shock in a high matter density environment. It is assumed to be due to collisionally excited $\mathrm{H}_{2}$ molecules heated by the shock.

An electronic bremsstrahlung hypothesis for the origin of the EGRET source (e.g., Bykov et al. 2000) is difficult to reconcile with the fact that the radio synchrotron, X-ray, and optical emissions are concentrated toward the rim of the remnant, whereas the EGRET source is located in the center. On the other hand, the optical emission seems to fade in regions where $\mathrm{CO}$ emission increases and where the MAGIC source is located (see Lasker et al. 1990). This perhaps indicates that the molecular material, which absorbs the optical radiation, is at the remnant's nearest side. Cornett et al. (1977) also argued that the molecular mass is located between us and the SNR. Troja et al. (2006) reached the same conclusions. The observed VHE $\gamma$-radiation may be due to $\pi^{0}$-decays from interactions between cosmic rays accelerated in IC 443 and the dense molecular cloud. A possible distance of this cloud from IC 443 might explain the steepness of the VHE $\gamma$-ray spectrum measured. As has been emphasized by Aharonian \& Atoyan (1996), the observed $\gamma$-rays can have a significantly different spectrum from that expected from the particle population at the source (the SNR shock).

The positions of 3EG J0617+2238, GeV J0617+2237, and MAGIC J0616+225 are all different from that of the pulsar wind nebula (PWN) CXOU J061705.3+222127 (Olbert et al. 2001; Bocchino \& Bykov 2001). The PWN is now colocated with a high-density molecular material region (Seta et al. 1998), which in addition is excited, as measured by a high $\operatorname{CO}(J=$ $2-1) / \mathrm{CO}(J=1-0)$ ratio. If the VHE $\gamma$-ray emission were re- lated to the PWN, one might expect some spatial overlap between the PWN and the $\gamma$-ray sources.

A complete coverage of the X-ray emission from the region was made with $X M M$ (Bocchino \& Bykov 2003), resulting in the detection of $12 \mathrm{X}$-ray sources with fluxes larger than $5 \times 10^{-14} \mathrm{ergs} \mathrm{cm}^{-2} \mathrm{~s}^{-1}$. None of these sources is spatially coincident with the MAGIC detection reported here. Rather, they are mostly located in the relatively small, $15^{\prime} \times 15^{\prime}$ region, for which the analysis of the 2MASS data reveals strong $2.2 \mu \mathrm{m}$ emission indicating interaction with a molecular cloud. However, the MAGIC source, uncorrelated with X-ray sources, is also cospatial with a region of high $2.2 \mu \mathrm{m}$ emission, but farther away from the shock.

In summary, the observation of IC 443 using the MAGIC telescope has led to the discovery of a new source of VHE $\gamma$-rays, MAGIC J0616+225, near the Galactic plane. A reasonably large data set was collected, and the spectrum of this source was measured up to energies of $1.6 \mathrm{TeV}$. The differential energy spectrum can be fit with a power law of slope $\Gamma=-3.1 \pm 0.3$. The coincidence of the VHE $\gamma$-ray source with SNR IC 443 leads us to posit this SNR as a natural counterpart, and although the mechanism responsible for the high-energy radiation remains yet to be clarified, a massive molecular cloud and $\mathrm{OH}$ maser emissions are located at the same sky position as that of MAGIC J0616+225, and they suggest that a nucleonic origin of the VHE $\gamma$-rays is possible.

We would like to thank the IAC for the excellent working conditions at the Observatory de los Muchachos in La Palma. The support of the German BMBF and MPG, the Italian INFN, and the Spanish CICYT is gratefully acknowledged. This work was also supported by ETH research grant TH 34/04 3 and the Polish MNiI grant 1P03D01028.

\section{REFERENCES}

Aharonian, F. A., \& Atoyan, A. M., 1996, A\&A, 309, 917

Aharonian, F. et al. 2006, ApJ, 636, 777

Albert, J. et al. 2006a, ApJ, 637, L41

. 2006b, ApJ, 638, L101

2006c, ApJ, 643, L53

2006d, preprint (astro-ph/0612385)

2007a, preprint (astro-ph/0702475)

2007b, preprint (arXiv:0705.3244)

Anykeev, V. B., Spiridonov, A. A., \& Zhigunov, V. B. 1991, Nucl. Instrum. Methods Phys. Res. A, 303, 350

Asaoka, I., \& Aschenbach, B. 1994, A\&A, 284, 573

Baixeras, C., et al. 2004, Nucl. Instrum. Methods Phys. Res. A, 518, 188

Baring, M. G., Ellison, D. C., Reynolds, S. P., Grenier, I. A., \& Goret, P. 1999, ApJ, 513, 311

Bartko, H., Goebel, F., Mirzoyan, R., Pimpl, W., \& Teshima, M. 2005, Nucl. Instrum. Methods Phys. Res. A, 548, 464

Bertero, M. 1989, Adv. Electron. Electron Phys., 75, 1

Bocchino, F., \& Bykov, A. M. 2000, A\&A, 362, L29 2001, A\&A, 376, 248 2003, A\&A, 400, 203

Bock, R. K., et al. 2004, Nucl. Instrum. Methods Phys. Res. A, 516, 511

Breiman, L. 2001, Machine Learning, 45, 5

Bretz, T., \& Wagner, R. 2003, Proc. 28th Int. Cosmic Ray Conf. (Tsukuba), 2947

Bykov, A. M., Bocchino, F., \& Pavlov, G. G. 2005, ApJ, 624, L41

Bykov, A. M., Chevalier, R. A., Ellison, D. C., \& Uvarov, Yu. A. 2000, ApJ, 538, 203

Claussen, M. J., Frail, D. A., Goss, W. M., \& Gaume, R. A. 1997, ApJ, 489, 143

Condon, J. J., Cotton, W. D., Greisen, E. W., Yin, Q. F., Perley, R. A., Taylor,

G. B., \& Broderick, J. J. 1998, AJ, 115, 1693

Cornett, R. H., Chin, G., \& Knapp, G. R. 1977, A\&A, 54, 889

Cortina, J. et al. 2005, Proc. 29th Int. Cosmic Ray Conf. (Pune), 5, 359

Dame, T. M., Hartmann, D., \& Thaddeus, P. 2001, ApJ, 547, 792

Domingo-Santamaria, E., et al. 2005, Proc. 29th Int. Cosmic Ray Conf. (Pune), 5,363

Esposito, J. A., Hunter, S. D., Kanbach, G., \& Sreekumar, P. 1996, ApJ, 461, 820
Fegan, D. J. 1997, J. Phys. G, 23, 1013

Fesen, R. A. 1984, ApJ, 281, 658

Fomin, V. P., Stepanian, A. A., Lamb, R. C., Lewis, D. A., Punch, M., \& Weekes, T. C. 1994, Astropart. Phys., 2, 137

Gaensler, B. M., Chatterjee, S., Slane, P. O., van der Swaluw, E., Camilo, F., \& Hughes, J. P. 2006, ApJ, 648, 1037

Gaisser, T. K., Protheroe, R. J., \& Stanev, T. 1998, ApJ, 492, 219

Gaug, M., et al. 2005, Proc. 29th Int. Cosmic Ray Conf. (Pune), 5, 375

Goebel, F., et al. 2007, Proc. 30th Int. Cosmic Ray Conf. (Merida), in press

Green, D. A. 2004, Bull. Astron. Soc. India, 32, 335

Hartman, R. C., et al. 1999, ApJS, 123, 79

Hewitt, J. W., Yusef-Zadeh, F., Wardle, M., Roberts, D. A., \& Kassim, N. E. 2006, ApJ, 652, 1288

Hillas, A. M. 1985, Proc. 19th Int. Cosmic Ray Conf. (La Jolla), 3, 445

Holder, J., et al. 2005, in AIP Conf. Proc. 745, High Energy Gamma-Ray Astronomy, ed. F. A. Aharonian, H. Völk, \& D. Horns (Melville: AIP), 275

Kaul, R. K. 2001, Bull. Astron. Soc. India, 29, 371

Keohane, J. W., Petre, R., Gotthelf, E. V., Ozaki, M., \& Koyama, K. 1997, ApJ, 484, 350

Khelifi, B., 2003, Ph.D. thesis, Univ. Caen/Basse-Normande, http://lpnp90.in2p3 .fr/\%7Ecat/Thesis/khelifi.pdf

Lamb, R. C., \& Macomb, D. J. 1997, ApJ, 488, 872

Lasker, B. M., Sturch, C. R., McLean, B. J., Russell, J. L., Jenkner, H., \& Shara, M. M. 1990, AJ, 99, 2019

Lessard, R. W., Buckley, J. H., Connaughton, V., \& Le Bohec, S. 2001, Astropart. Phys., 15, 1

Olbert, Ch. M., Clearfield, C. R., Williams, N. E., Keohane, J. W., \& Frail, D. A. 2001, ApJ, 554, L205

Paneque, D., Gebauer, H. J., Lorenz, E., \& Mirzoyan, R. 2004, Nucl. Instrum. Methods Phys. Res. A, 518, 619

Seta, M., et al. 1998, ApJ, 505, 286

Torres, D. F., Romero, G. E., Dame, T. M., Combi, J. A., \& Butt, Y. M. 2003, Phys. Rep., 382, 303

Troja, E., Bocchino, F., \& Reale, F. 2006, ApJ, 649, 258 